\title{
NEAR-EARTH ASTEROID (NEA) SCOUT
}

\author{
Leslie McNutt ${ }^{*}$, Les Johnson ${ }^{*}$, Peter Kahn ${ }^{\dagger}$, Julie Castillo-Rogez ${ }^{\dagger}$, \\ Andreas Frick ${ }^{\dagger}$ \\ "NASA Marshall Space Flight Center, Huntsville, Alabama, USA \\ ${ }^{\dagger}$ Jet Propulsion Laboratory, Pasadena, California, USA
}

\begin{abstract}
Near-Earth asteroids (NEAs) are the most easily accessible bodies in the solar system, and detections of NEAs are expected to grow exponentially in the near future, offering increasing target opportunities. As NASA continues to refine its plans to possibly explore these small worlds with human explorers, initial reconnaissance with comparatively inexpensive robotic precursors is necessary. Obtaining and analyzing relevant data about these bodies via robotic precursors before committing a crew to visit a NEA will significantly minimize crew and mission risk, as well as maximize exploration return potential.

The Marshall Space Flight Center (MSFC) and Jet Propulsion Laboratory (JPL) are jointly examining a potential mission concept, tentatively called 'NEAScout,' utilizing a low-cost platform such as CubeSat in response to the current needs for affordable missions with exploration science value. The NEAScout mission concept would be treated as a secondary payload on the Space Launch System (SLS) Exploration Mission 1 (EM-1), the first planned flight of the SLS and the second un-crewed test flight of the Orion Multi-Purpose Crew Vehicle (MPCV).
\end{abstract}

